\title{
Virulence factors and molecular characteristics of Shigella flexneri isolated from calves with diarrhea
}

\section{Zhen Zhu}

Hebei University of Engineering,

\section{Weiwei Wang}

Lanzhou Institute of Husbandry and Pharmaceutical Sciences of CAAS

\section{Mingze Cao}

Hebei University of Engineering,

\section{Qiqi Zhu}

Hebei University of Engineering,

\section{Tenghe Ma}

Hebei University of Engineering,

\section{Yongying Zhang}

Hebei University of Engineering,

\section{Guanhui Liu}

Hebei University of Engineering,

\section{Xuzheng Zhou}

Lanzhou Institute of Husbandry and Pharmaceutical Sciences of CAAS

\section{Bing Li}

Lanzhou Institute of Husbandry and Pharmaceutical Sciences of CAAS

\section{Yuxiang Shi}

Hebei University of Engineering,

Jiyu Zhang ( $\sim$ jiyuzhang123@163.com )

Lanzhou Institute of Husbandry and Pharmaceutical Sciences of CAAS

\section{Research Article}

Keywords: S. flexneri, virulence factors, MLST, MLVA, PFGE

Posted Date: March 9th, 2021

DOI: https://doi.org/10.21203/rs.2.22466/v5

License: (c) (i) This work is licensed under a Creative Commons Attribution 4.0 International License. Read Full License 
Version of Record: A version of this preprint was published at BMC Microbiology on July 16th, 2021. See the published version at https://doi.org/10.1186/s12866-021-02277-0. 


\section{Abstract \\ Background}

The natural hosts of Shigella are conventionally humans and other primates; however, the host range of Shigella has been shown to expand to many animals. Although Shigella is becoming a huge threat to animals, there is limited information on the genetic background of local strains. The purpose of this study was to assess the presence of virulence factors and the molecular characteristics of $S$. flexneri isolated from calves with diarrhea.

\section{Results}

Fifty-four $S$. flexneri isolates possessed four typical biochemical characteristics of Shigella. The prevalences of ipaH, virA, ipaBCD, ial, sen, set $1 A$, set1B and stx were $100 \%, 100 \%, 77.78 \%, 79.63 \%, 48.15 \%, 48.15 \%$ and 0 , respectively. MLVA based on 8 VNTR loci discriminated the isolates into 39 different MTs, PFGE based on Notl digestion divided the 54 isolates into 31 PTs, and MLST based on 15 housekeeping genes differentiated the isolates into 7 STs.

\section{Conclusion}

Our findings of this study have enriched our knowledge of the molecular characteristics of S. flexneri collected from diarrheal calves, which will be important for addressing clinical and epidemiological issues regarding Shigellosis.

\section{Background}

Shigellosis or blood dysentery is widespread in underdeveloped or developing regions with poor hygiene and limited access to clean drinking water, and has become a serious threaten to public health [1, 2]. Shigellosis is caused by nonmotile, facultative anaerobic Gram-negative bacilli of the Enterobacteriaceae family, includeing S. dysenteriae, S. flexneri, S. boydii, and S. sonnei [3-5]. Shigella species have highly effective on invasive systems that enable the bacteria to invade and multiply within the human intestinal epithelia, ultimately leading to severe inflammatory colitis, which is -named bacillary dysentery or shigellosis [4].

The various virulence factors located in the chromosome or large virulent inv plasmids is recognized as a crucial part that is related to the pathogenesis of shigellosis [6]. Moreover, these different virulence factors are associated with the colonization of intestinal cells and intracellular invasion, which may partly explain why there are various manifestations are detected in clinic, such as intestinal inflammatory responses and watery diarrhea [1].Bacterial cell-to-cell movement and dissemination within epithelial cells of intestine are allowed by $i p h H$ gene which encoded by chromosomal DNA and/or recombinant plasmid, while ia/ which are encoded by plasmid (invasion-associated locus) enables Shigella bacteria to penetrate into intestinal 
epithelial tissues $[7,8]$. The chromosomal genes set1A and set1B encode Shigella enterotoxin 1 (ShET-1) [9, 10], which is easily detected in all S. flexneri 2a. Shigella enterotoxin 2 which encoded by the gene sen, is located on a large plasmid associated with the virulence of Shigella and is found in most of Shigella of different serotypes and in enteroinvasive Escherichia coli (EIEC) $[11,12]$.It's recognized that ShET-1 and ShET-2, except for their enterotoxic activity, play significant roles in the transport of electrolytes and water in the intestine [12]. VirA located on large virulent plasmids has a great impact in intercellular spreading and invasion [13]. On the other hand, the type III secretion system (T3SS) is regarded as an important component for bacterial entry and is also composed of several proteins, including a needle-shaped oligomer anchored in the protein complex that connects the inner and outer bacterial membranes. The tip of the needle is an oligomer composed of the invasion plasmid antigens ipaB, ipaC, and ipaD [14-16]. Futhermore, the upstream $i p a B$ region is often used as a marker to detect the $i p a B C D$ gene.

The natural hosts of Shigella are conventionally humans and other primates [4]. However, it's reported that monkeys, rabbits, calves, fish, chickens and piglets are infected with Shigella as new hosts [4, 17-21]. In recent years, $S$. dysenteriae, S. flexneri, and S. sonnei are isolated from cows. Although Shigella is becoming a huge threat to animals, there is limited information on the genetic background of isolated strains. Therefore, aim to indentify molecular genotype and determine the genetic relatedness diversity of local $S$. flexneri strains, we performed the test via the methods of MLST, MLVA and PFGE.

\section{Results}

\section{Biochemical characterization}

A total of 54 S. flexneri of six serotypes, including $1 a(n=5), 2 a(n=26), 2 b(n=4), 4 a(n=6), 6(n=8)$, and Xv $(n=5)$, were analyzed in this study. Based on the results of the biochemical reaction assays, we observed that all 54 S. flexneri isolates possessed 4 typical Shigella biochemical characteristics (Table 1). Among these BTs, BT4 (the ability to ferment glucose, mannitol, arabinose, and melibiose) was the predominant biotype, accounting for $70.37 \%$ (38/54) of all BTs. Furthermore, BT4 was widely found in each serotype, except serotype 6 . S. flexneri 2a was distributed among all four biochemical phenotypes and mainly in BT4 $(22 / 26,84.62 \%)$. However, the other five serotype strains only had one or two biochemical phenotypes.

\section{Virulence factors}

The frequencies of the virulence factor profiles in the $S$. flexneri isolates are listed in Fig 1 . A total of seven virulence factors were detected in those isolates, including ipaH (100\%), virA (100\%), ipaBCD (92.59\%), ial (77.78\%), sen (79.63), set1A (48.15\%) and set1B (48.15\%). None of the studied strains possessed the stx gene. The Shigella enterotoxin genes set1A and set1B were only present in $S$. flexneri $2 a$, and all of these serotype isolates were positive for these two genes.

Regarding the differences in the distributions of the virulence factors, the $54 \mathrm{~S}$. flexneri isolates fell into seven gene profile types (VT) (Table 2). Among these VTs, VT4 (positive for ipaH, virA, ipaBCD, ial, sen) and VT6 (positive for ipaH, virA, ipaBCD, ial, sen, set1A, set1B) were the most common, accounting for $29.63 \%$ 
and $44.44 \%$ of all VTs, respectively. Furthermore, $92.59 \%$ of the isolates carried two or more virulence factors. In addition, the virulence factor types were associated with the $S$. flexneri serotype. VT1 was only found in $4 \mathrm{a}$, and VT4 was present in isolates from each serotype, except 2a. S. flexneri 2a major belonged to VT6 $(24 / 26,92.31 \%)$.

\section{MLST-based genotype analysis}

MLST was performed to analyze the genotypic diversity of $S$. flexneri isolates based on 15 housekeeping genes. The 54 isolates were divided into seven STs, including ST68, ST100, ST103, ST120, ST124, ST135 and ST227. Among them, ST227 was novel, while the six other STs have previously been reported. These seven STs belonged to several clonal complexes (CCs): CC10 (ST100 and ST103), CC26 (ST68), and others (ST120, ST124, ST135 and ST227). The clustering tree (Fig 2) based on the MLST data showed that ST68 was a singleton type and that the other six STs contained two or more isolates. The most common ST was ST100 ( $n=33,61.11 \%)$, including isolates of serotypes 1a, 2a, and Xv. All the isolates of ST124 and ST227 belonged to S. flexneri 6 and 4a, respectively. The cluster tree indicated that isolates belonging to the same serotype closely clustered based on the province of isolation. In addition, according to the minimum spanning tree (MST) based on the allele, it was found that ST100, ST120 and ST135 had closer relationships and only differed in aspC, while ST68, ST124 and ST227 were very different from the other STs (Fig 3).

\section{MLVA-based genotype analysis}

MLVA based on eight VNTR loci was performed to further characterize the isolated $S$. flexneri strains. The copy numbers of the eight VNTR loci are listed in Fig 4. Overall, the 54 isolates based on the unique MLVA profiles were discriminated into 39 different MLVA types (MTs). Among them, twenty-eight MTs belonged to the singleton type, and the other ten MTs contained no more than three isolates. The MLVA cluster tree of the isolates showed that they were divided into five clusters, designated A to E, with a low coefficient of similarity from $20 \%-60 \%$ (Fig 4). Each cluster was further divided into many subclusters. MLVA can cluster different serotype strains separately and distinguish between the same serotype strains. The main cluster, cluster C, was observed clustering $S$. flexneri 2a isolates and further divided into 15 MTs. Additionally, clusters A (except GBSF1502176), D and E only clustered the Xv, 2b, and 6 serotype strains, respectively. The results showed differences based on the geographical origin and time span in the same serotype.

\section{PFGE-based genotype analysis}

The genotypes and genetic relatedness diversity of the $54 \mathrm{~S}$. flexneri isolates were assessed by PFGE. Nottdigested S. flexneri chromosomal DNA generated 31 reproducible unique PFGE patterns (PTs), each with 1116 bands (Fig 5). Eleven patterns were represented by more than one isolate, with PT20 $(n=8)$ containing the most isolates, followed by PT18 $(n=5)$. The dendrogram of $S$. flexneri isolates showed low similarity 
(40\%-60\%) and could be classed into three gross clusters on the basis of their serotypes: clusters A, B and C. Isolates belonging to the same serotype but recovered in different years showed clear relatedness, as indicated by their grouping in the same clusters. The majority of serotype $2 a$ isolates, with the exception of isolate QYSF1511395, grouped together in cluster B. The QYSF1511395 strain isolated from Qinghai Province clustered independently in cluster $\mathrm{C}$. Isolates $1 \mathrm{a}, 2 \mathrm{~b}$ and $\mathrm{Xv}$ clustered into cluster $\mathrm{B}$ and were closely related to the serotype $2 \mathrm{a}$ isolates. However, the isolates of serotypes $4 \mathrm{a}$ and 6 were assigned to cluster A with a relatively close relationship, but different serotype strains clustered separately.

\section{Discussion}

Shigella is an important invasive enteric infectious pathogen known for its sporadic, epidemic and pandemic spread [3], which is still a landmark cause of inflammatory diarrhea and dysentery, posing a serious challenge to public health, especially tracked in most middle-income countries and regions with substandard hygiene and poor qualitily water supplies [22]. All four kinds of Shigella can cause shigellosis, but $S$. flexneri is the most common bacterial preparation in shigellosis [23]. The traditional hosts of this pathogen are limited in primates; however, the range of host has been extended to many animals in recent decades [4]. The symptoms of shigellosis in human reported included: diarrhea (100\%), headache (100\%), fever (100\%), nausea (99\%), abdominal cramping (97\%), vomiting (95\%), and bloody stools (51\%), however, it is not clear on animals [24]. Better understanding of the hosts of Shigella is needed to assess its potential effects on animal health, otherwise, it's a challenge for preventing the disease caused by Shigella.

Pathogenesis of Shigella is contributed to the organism's ability to invade, replicate and spread intercellularly within the colonic epithelium. Pathogenic factors are the reasons that pathogenic Shigella invades intestinal epithelial cells, leading to dysentery and other intestinal clinical symptoms in the host [25], and its pathogenesis is often multifactorial and coordinated [26]. Virulence factor has become an important indicator of pathogenic bacteria.

Based on the detection of virulence factors, the Shigella isolates used in the present study had vast genetic diversity. Our results showed that $i p a H$ and virA were found in each strain. Arabshahi et al. showed a similar result that $i p a H$ was present in all Shigella isolates, and virA was harbored by $88.9 \%$ (8/9) of S.flexneri [27]. In agreement with the previous study which demonstrating that $i p a H$ is carried by all four Shigella species as well as by enteroinvasive E. coli (EIEC). Multiple copies (ipaH1.4, ipaH2.5, ipaH4.5, ipaH7.8 and ipaH9.8) on large plasmids and chromosomes may explain why the $i p a H$ gene tested positive in all isolates.

Therefore, as a diagnostic tool for detecting Shigella, the $i p a H$ gene is often an appealing target, even in the absence of the plasmid [28]. VirA was initially thought to be an invasion in Shigella; however, structural analysis showed that VirA lacks lacked papain like protease activity to promote tubulin division. VirA belongs to the GTPase activating protein family, which is involved in the cleavage of single membrane into vacuole. Previous studies have shown that VirA is often present in Shigella, and it is an important terminal point for bacteria to invade host cells and nucleate actin at one end of bacteria [9, 29].

Expert opinion suggested that T3SS is essential for host cell invasion and intracellular survival among those virulence factors, whereas IpaB, IpaC, and IpaD are key factors of virulent Shigella $[9,30,31]$. Unlike 
the $i p a H$ gene, the $i a l$ gene is not common. The ipaH gene is only located on the inv plasmid, and compared with chromosome gene, the stability of IPAH plasmid to storage/subculturing is poor [6-8]. Our results show that the ial gene has a high invasiveness in the isolates studied. Therefore, it should be noted that the $i a /$ gene is involved in the invasion of intestinal cells and that the higher positive rate of this gene in $S$. flexneri might indicate stronger aggressiveness.

The Shigella enterotoxins ShET-1 and ShET-2 as alter electrolyte and water transport for small intestine can cause diarrhea and dehydration [22]. ShET-1 is located on chromosomes encoding $\operatorname{set} 1$ (A and B subunit) genes, which is almost exclusively found in several $S$. flexneri serotype 2 isolates and is rarely found in other serotypes [32]. Consistent with previous studies, our study showed that $\operatorname{set} 1 A$ and $\operatorname{set} 1 B$ were only detected in the $S$. flexneri 2a strain. It is known that the plasmid encoding ShET-2 (encoded by sen) is an enterotoxin hemolysin that causes an inflammatory response during the invasion of Shigella [12, 22]. It's reported that there is a close relationship among sen, set enterotoxins and bloody diarrhea [22]. And it implied that sen and set enterotoxins is the pathogentic factor of bloody diarrhea. However, unlike ShET-1, ShET-2 could be harbored by other species of Shigella.

Molecular characterization of strains is significant for epidemiological studies. However, rare reports are available to systematically understand the molecular characteristics of $S$. flexneri isolated from animals. Recently, several useful genotyping tools with higher discriminatory power than traditional tests, which containing MLST [33], PFGE [34] and MLVA [35], have been applied to explore and analysis the characteristic of Shigella isolates. The method of analysis on relationship of phylogeny as an important part to support strain isolation is based on the difference of strain genetics.

MLST is an important source of sequence data for relative genetics, providing a tool for exploring molecular evolutionary methods among bacteria [36]. With the key elements of 15 housekeeping genes and analysis of the EcMLST database, the advantage of MLST is that the comparison of data from different laboratories. Our results suggested that the predominant ST was ST 100, which has before been found in human $S$. flexneri isolates $[37,38]$. To be specific, isolates belonging to the same serotype often showed one ST type, indicating the low discriminative ability in closely related strains within a specific serotype due to the high sequence conservation of the housekeeping genes.

Compared with the MLST profiles, MLVA and PFGE may be forceful tools that can provide a satisfactory level of discrimination. Whereas the function of MLVA in phylogenetic analysis of different bacterial species or serotypes are poorly targeted [39]. Nevertheless, MLVA is an ordinarily used typing tool that has been used for establishing genetic relatedness and performing phylogenetic analysis among strains of monomorphic species. In our study, with approximately $20 \%$ similarity, the $54 \mathrm{~S}$. flexneri isolates were divided into 39 different MTs and clustered into 5 groups. Previous studies also showed the high resolving power of MLVA in closely related strains [40-42]. Though applied in a limited collection of $S$. flexneri isolates, this study indicates the high discriminatory power of the MLVA method for subtyping strains with the same serotype. 
With the strong function and widespread use, PFGE is also an applicable typing tool available in the laboratory for discriminating several enteric bacteria, such as Shigella. PFGE has a high degree of intra- and interlaboratory reproducibility when standardized protocols are followed [43]. Thirty-one low homophyly and unique PFGE patterns confirmed the existence of diverse $S$. flexneri clones and the usefulness of PFGE in local epidemiological studies.

\section{Conclusion}

This study demonstrated that spontaneous prevalent $S$. flexneri in cows shelter the same virulence factors as the prevalent isolates in humans. Therefore, these isolates are a potential threat to public safety. To systematically understand S. flexneri, PFGE, MLVA and MLST methods were applied to hereditary characterize the 54 isolates. MLVA based on 8 VNTR loci discriminated the 54 isolates into 39 different MTs, PFGE based on Notl digestion ambiguous the 54 isolates into 31 PTs, while MLST based on 15 housekeeping genes differentiated the 54 isolates into 7 STs, with 1 ST (ST227) being novel. Although MLST provided suitable discrimination in S. flexneri subtyping, PFGE and MLVA might both exhibit a higher discriminatory ability. Overall, the data from this study will provide a useful typing resource, which will provide a scientific basis for addressing clinical and epidemiological issues regarding $S$. flexneri.

\section{Methods}

\section{Bacterial isolates and bacteriological examination}

Animal-based active surveillance was conducted in 3321 diarrhea calves from five provinces (Gansun, Shanxi, Qinghai, Xinjiang and Tibet) in northwestern China from 2014 to 2016. All of the isolates were collected directly from fresh stool samples following plating on Salmonella-Shigella (SS) selective agar and confirming on MacConkey (MAC) agar with $37^{\circ} \mathrm{C}$ for $24 \mathrm{~h}$. The colorless, semitransparent, smooth, and moist circular plaques were considered presumptive Shigella for biochemical confirmation. Biochemical tests were performed on S. flexneri using API20E test strips (bioMerieux Vitek, Marcy-l' Etoile, France), and the serotype was tested by a commercially available agglutinating antibody kit (Denka Seiken, Tokyo, Japan) according to the manufacturers' recommendations. The information of $S$. flexneri isolates in this study were list in Fig 2, Fig 4, Fig5.

\section{Preparation of DNA templates}

The DNA templates for PCR (virulence factors, MLST, MLVA) were directly extracted from bacterial colonies using the boiled lysate method, as previous report [44].

\section{Detection of virulence factors}

All 54 strains were tested by PCR for the presence of 8 virulence-associated genes, including ipaH, ipaBCD, virA, ial, stX, set1 $A$, set1B, and sen according to published procedures [45-47]. PCR reactions were performed 
according to published protocols and the primers sequences were listed in Table S1.

\section{Multilocus sequence typing (MLST)}

All isolates were subjected to MLST according to the protocols described in the EcMLST database (http://www.shigatox.net/ecmlst). The PCR products were bidirectionally sequenced, and the sequences of the 15 housekeeping genes were edited by using SeqMan 7.0. Each unique allele was assigned a different number, and the allelic profile (string of fifteen allelic loci) was used to define each isolate's sequence type (ST) [48]. Clustering and minimum spanning tree (MST) analyses were used to infer relationships among the isolates using the fingerprint analysis software BioNumerics (version 7.1).

\section{Multiple-locus variable number tandem repeat analysis (MLVA)}

MLVA analysis of 8 VNTR loci (SF3, SF4, SF6, SF7, SF8, SF9, SF10 and SF25) was performed using a previously described method [35]. The forward primer for each primer set was labelled at its 5 ' end with an ABI compatible dye: HEX, 6'-FAM, TAMRA, and ROX (Table S2). In these cases, the loci were individually amplified, with each $20 \mu \mathrm{L}$ PCR mixture containing $1 \mu \mathrm{L}$ each primer, $1 \mu \mathrm{L}$ DNA template, $10 \mu \mathrm{L}$ Taq MasterMix (Takara, Japan) and deionized water to a final volume of $20 \mu \mathrm{L}$. PCR was performed with a denaturing step of $94^{\circ} \mathrm{C}$ for $5 \mathrm{~min}$, followed by 30 cycles of amplification at $94^{\circ} \mathrm{C}$ for $30 \mathrm{~s}, 55^{\circ} \mathrm{C}$ for $45 \mathrm{~s}$, and $72^{\circ} \mathrm{C}$ for $45 \mathrm{~s}$ and a final extension at $72^{\circ} \mathrm{C}$ for $5 \mathrm{~min}$ at the final step.

The PCR products were analyzed by capillary electrophoresis on an ABI Prism 3730 XL Genetic Analyzer with the GeneScan 500 LIZ Size Standard as previously described [49]. The number of repeat units for each allele was converted from the length of the amplicon. The copy number of each VNTR locus was subjected to cluster analysis using the MST algorithm and the categorical coefficient provided in the BioNumerics software. Each unique allelic string was designated a unique MLVA type (MT). A dendrogram was constructed by UPGMA clustering based on categorical coefficient analysis [35,50].

\section{Pulsed field gel electrophoresis (PFGE)}

DNA fingerprinting was performed by PFGE with the restriction enzyme Notl (TaKaRa; Japan) according to the international standards set by the CDC. PFGE images were photographed with a Universal Hood II (BioRad; USA) and analyzed with BioNumerics using the Dice similarity coefficient, unweighted pair-group method with the arithmetic mean (UPGMA) and $1.0 \%$ band position tolerance. A PFGE type (PT) was defined as a pattern with one or more DNA bands different from other patterns.

\section{Abbreviations}

VTs: Virulence gene profile types; BTs: MLVA: Multiple-locus variable number tandem repeat analysis; VNTR: Variable Number of Tandem Repeats; PFGE: Pulsed-field gel electrophoresis; MLST: Multilocus sequence 
typing.

\section{Declarations}

\section{Ethics approval and consent to participate}

The study was carried out in compliance with the ARRIVE guidelines and gained ethical approval from the Ethics Committee of Lanzhou Institute of Husbandry and Pharmaceutical Sciences of CAAS. Our study was conducted according to the Animal Administration and Ethics Committee of Lanzhou Institute of Husbandry and Pharmaceutical Sciences of CAAS (Permit No. SYXK-2014-021). And we gained informed consent from the owners of the animals for them to be used in the study.

\section{Consent for publication}

Not Applicable.

\section{Availability of data and material}

The data supporting the findings of this study are contained within the manuscript.

\section{Competing interests}

The authors declare that they have no competing interests.

\section{Funding}

The design of the study including collection, analysis, and interpretation of data was supported by the grants from the National Natural Science Foundation of China (No:31872520) and Drug Development and Clinical Drug Use Posts of National Beef Yak Industry Technical System (No: CARS-37); And the writing and submission the manuscript was supported by the Natural Science Foundation of Hebei Province (No: C2019402114) and a grant (2018SKLID308) from the State Key Laboratory for Infectious Disease Prevention and Control (China CDC).

\section{Authors' contributions}

Z.Z. and J.Y.Z. designed the study; Z.Z., M.Z.C., T.H.M., Y.X.S., X.Z.Z., G.H. L and B.L. generated and provided the dataset; Z.Z., Q.Q.Z., Y.Y.Z. and W.W.W. performed the experiments, analyzed the data, and wrote the manuscript. All authors have read and approved the final manuscript

\section{Acknowledgements}

The authors thank all the State Key Laboratory for Infectious Disease Prevention and Control (China CDC) for providing funding and technical support.

\section{Authors' information}


Key Laboratory of New Animal Drug Project of Gansu Province, Key Laboratory of Veterinary Pharmaceutical Development of Ministry of Agriculture, Lanzhou Institute of Husbandry and Pharmaceutical Sciences of CAAS, Jiangouyan, Qilihe District, Lanzhou, PR China

College of Life Science and Food Engineering, Hebei University of Engineering, Hanshan District, Handan, China

\section{References}

1. Dutta S, Jain P, Nandy S, Matsushita S, Yoshida S. Molecular characterization of serologically atypical provisional serovars of Shigella isolates from Kolkata, India. J Med Microbiol. 2014; 63(Pt 12):1696703.

2. Soltan Dallal MM, Ranjbar R, Pourshafie MR. The study of antimicrobial resistance among Shigella flexneri strains isolated in Tehran, Iran. J Pediatr Infect Dis. 2011;6:125-9.

3. Ranjbar R, Bolandian M, Behzadi P. Virulotyping of Shigella spp. isolated from pediatric patients in Tehran, Iran. Acta Microbiol Immunol Hung. 2017;64(1):71-80.

4. Shi R, Yang X, Chen L, Chang HT, Liu HY, Zhao J, et al. Pathogenicity of Shigella in chickens. PLoS One. 2014; 9(6):e100264.

5. Ranjbar R, Behnood V, Memariani H, Najafi A, Moghbeli M, Mammina C. Molecular characterisation of quinolone-resistant Shigella strains isolated in Tehran, Iran. J Glob Antimicrob Resist. 2016;5:26-30.

6. Shen Y, Qian H, Gong J, Deng F, Dong C, Zhou L, et al. High prevalence of antibiotic resistance and molecular characterization of integrons among Shigella isolates in Eastern China. Antimicrob Agents Chemother. 2013;57(3):1549-51.

7. Phantouamath B, Sithivong N, Insisiengmay S, Ichinose Y, Higa N, Song T, et al. Pathogenicity of Shigella in healthy carriers: a study in Vientiane, Lao People's Democratic Republic. Jpn J Infect Dis. 2005;58(4):232-4.

8. Ashida H, Sasakawa C. Shigella IpaH Family Effectors as a Versatile Model for Studying Pathogenic Bacteria. Front Cell Infect Microbiol. 2016;5:100.

9. Schroeder GN, Hilbi H. Molecular pathogenesis of Shigella spp.: controlling host cell signaling, invasion, and death by type III secretion. Clin Microbiol Rev. 2008;21(1):134-56.

10. Sousa MÂ, Mendes EN, Collares GB, Péret-Filho LA, Penna FJ, Magalhães PP. Shigella in Brazilian children with acute diarrhoea: prevalence, antimicrobial resistance and virulence factors. Mem Inst Oswaldo Cruz. 2013;108(1):30-5.

11. Farfán MJ, Toro CS, Barry EM, Nataro JP. Shigella enterotoxin-2 is a type III effector that participates in Shigella-induced interleukin 8 secretion by epithelial cells. FEMS Immunol Med Microbiol. 2011;61(3):332-9.

12. da Cruz CB, de Souza MC, Serra PT, Santos I, Balieiro A, Pieri FA, et al. Virulence factors associated with pediatric shigellosis in Brazilian Amazon. Biomed Res Int. 2014;2014:539697. 
13. Zaidi MB, Estrada-García T. Shigella: a highly virulent and elusive pathogen. Curr Trop Med Rep. 2014;1:81-7.

14. Büttner D, Bonas U. Who comes first? How plant pathogenic bacteria orchestrate type III secretion. Curr Opin Microbiol. 2006;9(2):193-200.

15. Faruque SM, Khan R, Kamruzzaman M, Yamasaki S, Ahmad QS, Azim T, et al. Isolation of Shigella dysenteriae type 1 and $\mathrm{S}$. flexneri strains from surface waters in Bangladesh: comparative molecular analysis of environmental Shigella isolates versus clinical strains. Appl Environ Microbiol. 2002;68(8):3908-13.

16. Marteyn B, Gazi A, Sansonetti P. Shigella: a model of virulence regulation in vivo. Gut Microbes. 2012;3(2):104-20.

17. Onyango DM, Wandili S, Kakai R, Waindi EN. Isolation of Salmonella and Shigella from fish harvested from the Winam Gulf of Lake Victoria, Kenya. J Infect Dev Ctries. 2009;3(2):99-104.

18. Priamukhina NS, Kilesso VA, Tikhomirov ED. Animal carriers of Shigella and their possible epidemiological importance. Zh Mikrobiol Epidemiol Immunobiol. 1984;(11):20-4.

19. Maurelli AT, Routh PR, Dillman RC, Ficken MD, Weinstock DM, Almond GW, et al. Shigella infection as observed in the experimentally inoculated domestic pig, Sus scrofa domestica. Microb Pathog. 1998;25(4):189-96.

20. Zhu Z, Cao M, Zhou X, Li B, Zhang J. Epidemic characterization and molecular genotyping of Shigella flexneri isolated from calves with diarrhea in Northwest China. Antimicrob Resist Infect Control. 2017;6:92.

21. Zhu Z, Shi Y, Zhou X, Li B, Zhang J. Molecular characterization of fluoroquinolone and/or cephalosporin resistance in Shigella sonnei isolates from yaks. BMC Vet Res. 2018;14(1):177.

22. Gu B, Fan W, Qin T, Kong X, Dong C, Tan Z, et al. Existence of virulence factors in clinical Shigella sonnei isolates from Jiangsu Province of China: a multicenter study. Ann Transl Med. 2019;7(14):305.

23. Sangeetha AV, Parija SC, Mandal I, Krishnamurthy S. Clinical and microbiological profiles of shigellosis in children. J Health Popul Nutr. 2014; 32(4):580-6.

24. Ranjbar R, Hosseini MJ, Kaffashian AR, et al. An outbreak of shigellosis due to Shigella flexneri serotype 3a in a prison in Iran. Arch Iran Med. 2010, 13(5):413-6.

25. Ashida H, Ogawa M, Mimuro H, Kobayashi T, Sanada T, Sasakawa C. Shigella are versatile mucosal pathogens that circumvent the host innate immune system. Curr Opin Immunol. 2011;23(4):448-55.

26. Qu M, Zhang X, Liu G, Huang Y, Jia L, Liang W, et al. An eight-year study of Shigella species in Beijing, China: serodiversity, virulence factors, and antimicrobial resistance. J Infect Dev Ctries. 2014;8(7):9048.

27. Arabshahi S, Novinrooz A, Ranjbar R, et al. Molecular characterization of Shigella species isolated from diarrheal patients in Tehran, Iran: phylogenetic typing and its association with virulence gene profiles and a novel description of Shigella invasion associated locus. Eur J Clin Microbiol Infect Dis. 2020, 39(9):1727-1737. 
28. Vu DT, Sethabutr O, Von Seidlein L, Tran VT, Do GC, Bui TC, et al. Detection of Shigella by a PCR assay targeting the ipaH gene suggests increased prevalence of shigellosis in Nha Trang, Vietnam. J Clin Microbiol. 2004;42(5):2031-5.

29. Yaghoubi S, Ranjbar R, Dallal MMS, Fard SY, Shirazi MH, Mahmoudi M. Profiling of Virulenceassociated Factors in Shigella Species Isolated from Acute Pediatric Diarrheal Samples in Tehran, Iran. Osong Public Health Res Perspect. 2017;8(3):220-226.

30. Parsot C. Shigella type III secretion effectors: how, where, when, for what purposes? Curr Opin Microbiol. 2009;12(1):110-6.

31. Mattock E, Blocker AJ. How Do the Virulence Factors of Shigella Work Together to Cause Disease? Front Cell Infect Microbiol. 2017;7:64.

32. Roy S, Thanasekaran K, Dutta Roy AR, Sehgal SC. Distribution of Shigella enterotoxin genes and secreted autotransporter toxin gene among diverse species and serotypes of shigella isolated from Andaman Islands, India. Trop Med Int Health. 2006;11(11):1694-8.

33. Cao Y, Wei D, Kamara IL, Chen W. Multi-Locus Sequence Typing (MLST) and Repetitive Extragenic Palindromic Polymerase Chain Reaction (REP-PCR), characterization of shigella spp. over two decades in Tianjin China. Int J Mol Epidemiol Genet. 2012;3(4):321-32.

34. Xia S, Xu B, Huang L, Zhao JY, Ran L, Zhang J, et al. Prevalence and characterization of human Shigella infections in Henan Province, China, in 2006. J Clin Microbiol. 2011; 49(1):232-42.

35. Wang YW, Watanabe H, Phung DC, Tung SK, Lee YS, Terajima J, et al. Multilocus variable-number tandem repeat analysis for molecular typing and phylogenetic analysis of Shigella flexneri. BMC Microbiol. 2009; 9:278.

36. Hu Y, Xi Z, Liu X, Wang J, Guo Y, Ren D, et al. Identification and molecular characterization of Wolbachia strains in natural populations of Aedes albopictus in China. Parasit Vectors. 2020; 13(1):28.

37. Li S, Sun Q, Wei X, Klena JD, Wang J, Liu Y, et al. Genetic characterization of Shigella flexneri isolates in Guizhou Province, China. PLoS One. 2015;10(1): e0116708.

38. Cui X, Wang J, Yang C, Liang B, Ma Q, Yi S, et al. Prevalence and antimicrobial resistance of Shigella flexneri serotype 2 variant in China. Front Microbiol. 2015; 6:435.

39. Cheng T, Shi X, Yong W, Wang J, Xie G, Ding J. Molecular typing of Shigella sonnei isolates circulating in Nanjing, China, 2007-2011. J Infect Dev Ctries. 2014;8(12):1525-32.

40. Filliol-Toutain I, Chiou CS, Mammina C, et al. Global Distribution of Shigella sonnei Clones. Emerg Infect Dis. 2011, 17(10):1910-2.

41. Ranjbar R, Memariani M, Memariani H. Diversity of Variable Number Tandem Repeat Loci in Shigella Species Isolated from Pediatric Patients. Int J Mol Cell Med. 2015, 4(3):174-81.

42. Ranjbar R, Memariani M. Multilocus variable-number tandem-repeat analysis for genotyping of Shigella sonnei strains isolated from pediatric patients. Gastroenterol Hepatol Bed Bench. 2015, 8(3):225-32.

43. Ribot EM, Fair MA, Gautom R, Cameron DN, Hunter SB, Swaminathan B, et al. Standardization of pulsed-field gel electrophoresis protocols for the subtyping of Escherichia coli 0157:H7, Salmonella, and Shigella for PulseNet. Foodborne Pathog Dis. 2006;3(1):59-67. 
44. Ahmed AM, Shimamoto T, Shimamoto $T$, et al. Molecular characterization of multidrug-resistant avian pathogenic Escherichia coli isolated from septicemic broilers. Int J. Med Microbiol. 2013, 303:475-483.

45. Das A, Natarajan M, Mandal J. The emergence of quinolone resistant Shigella sonnei, Pondicherry, India. PLoS One, 2016, 11(8):e0160290.

46. Vargas M, Gascon J, Jimenez De Anta MT, Vila J. Prevalence of Shigella enterotoxins 1 and 2 among Shigella strains isolated from patients with traveler's diarrhea. J Clin Microbiol. 1999;37(11):3608-11.

47. Faruque SM, Khan R, Kamruzzaman M, Yamasaki S, Ahmad QS, Azim T, et al. Isolation of Shigella dysenteriae type 1 and $\mathrm{S}$. flexneri strains from surface waters in Bangladesh: comparative molecular analysis of environmental Shigella isolates versus clinical strains. Appl Environ Microbiol. 2002;68(8):3908-13.

48. Schaumburg F, Alabi AS, Kaba H, et al. Molecular characterization of Shigella spp. from patients in Gabon 2011-2013. Trans R Soc Trop Med Hyg. 2015,109(4):275-9.

49. Liang SY, Watanabe H, Terajima J, et al. Multilocus Variable-Number Tandem Repeat Analysis for Molecular Typing of Shigella sonnei. J Clin Microbiol. 2007, 45(11): 3574-3580.

50. Hyytia-Trees E, Smole SC, Fields PA, et al. Second generation subtyping: a proposed PulseNet protocol for multiple-locus variable-number tandem repeat analysis of Shiga toxin-producing Escherichia coli 0157 (STEC 0157). Foodborne pathog dis.2006, 3:118-131.

\section{Tables}

Table 1 Biochemical characteristics of S.flexneri isolates.

\begin{tabular}{|c|c|c|c|c|c|c|c|c|}
\hline & \multirow[t]{2}{*}{ Biotype } & \multirow{2}{*}{$\begin{array}{l}\text { Total } \\
(\mathrm{n}=54)\end{array}$} & \multicolumn{5}{|c|}{ Isolates } & \multirow[b]{2}{*}{$\begin{array}{c}\mathrm{Xv} \\
(\mathrm{n}=5)\end{array}$} \\
\hline & & & $\begin{array}{c}1 \mathrm{a} \\
(\mathrm{n}=5)\end{array}$ & $2 a(n=26)$ & $\begin{array}{c}2 \mathrm{~b} \\
(\mathrm{n}=4)\end{array}$ & $4 a(n=6)$ & $6(n=8)$ & \\
\hline BT1 & $\begin{array}{l}\text { glucose+, mannose+, } \\
\text { arabinose-, melibiose+ }\end{array}$ & $2(3.70 \%)$ & 0 & $2(7.69 \%)$ & 0 & 0 & 0 & 0 \\
\hline BT2 & $\begin{array}{l}\text { glucose+, mannose+, } \\
\text { arabinose+, melibiose- }\end{array}$ & 9 (16.67\%) & 0 & $1(3.85 \%)$ & 0 & 0 & $8(100 \%)$ & 0 \\
\hline BT3 & $\begin{array}{l}\text { glucose+, mannose-, } \\
\text { arabinose+, melibiose+ }\end{array}$ & $5(9.26 \%)$ & 0 & $1(3.85 \%)$ & 0 & $4(66.67 \%)$ & 0 & 0 \\
\hline BT4 & $\begin{array}{l}\text { glucose+, mannose+, } \\
\text { arabinose+, melibiose+ }\end{array}$ & $38(70.37 \%)$ & $\begin{array}{c}5 \\
(100 \%) \\
\end{array}$ & $22(84.62 \%)$ & $4(100 \%)$ & $2(33.33 \%)$ & 0 & $5(100 \%)$ \\
\hline
\end{tabular}

Table 2 Statistical the rate of each virulence genes types in S.flexneri isolates. 


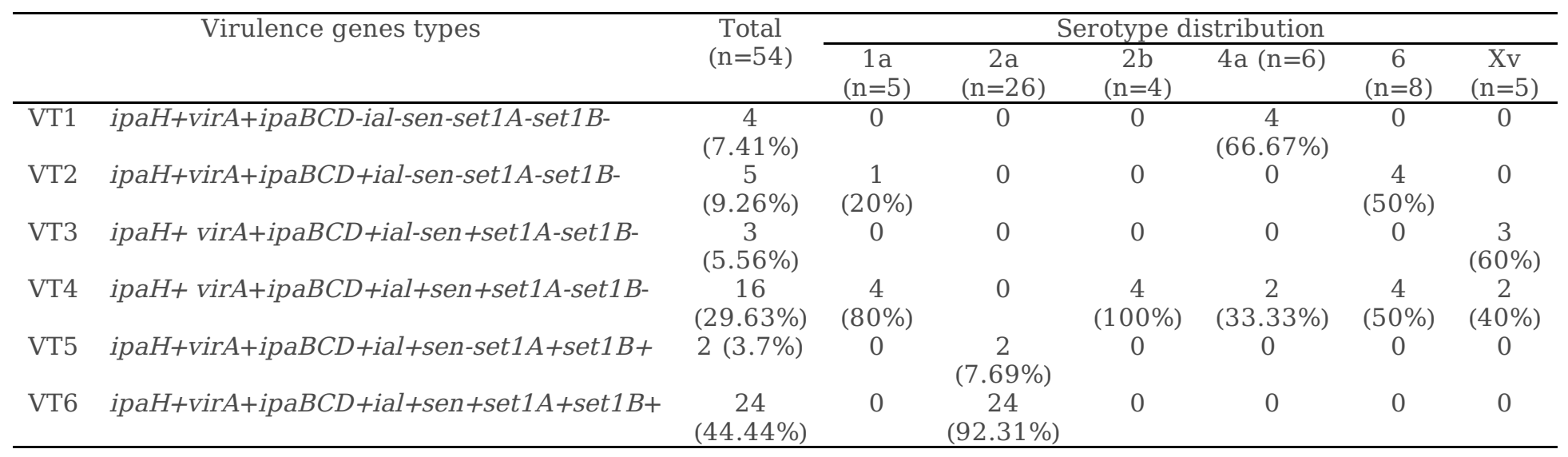

\section{Figures}




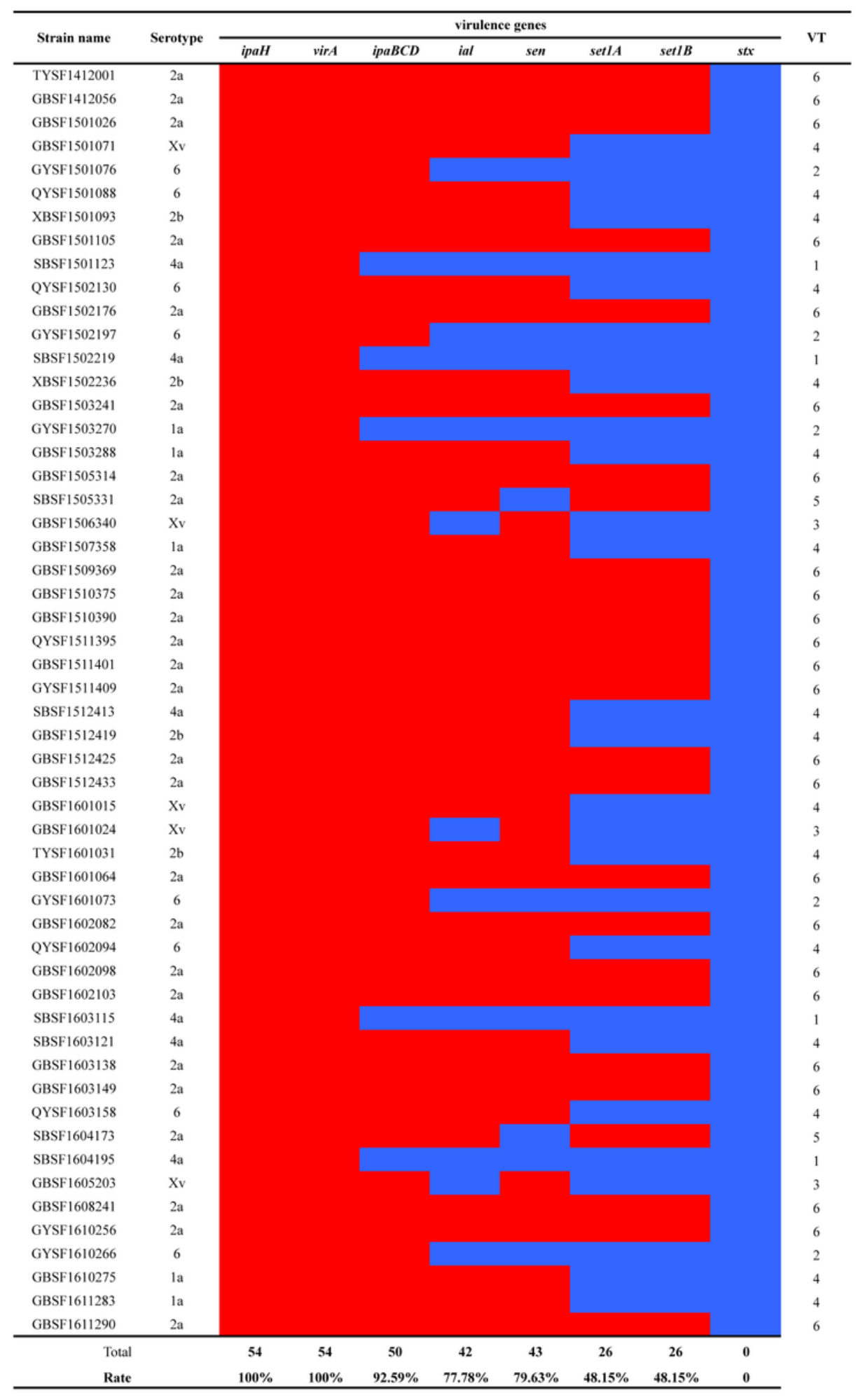

Figure 1

Statistical analysis of the presence of virulence factors in S. flexneri isolates. Red=present; Blue=absent. 
Figure 2

MLST clustering tree of S. flexneri isolates isolated from 2014 to 2016 from diarrhea calves. The 54 isolates were analyzed using a 15 allele MLST as described in the Materials and Methods. 


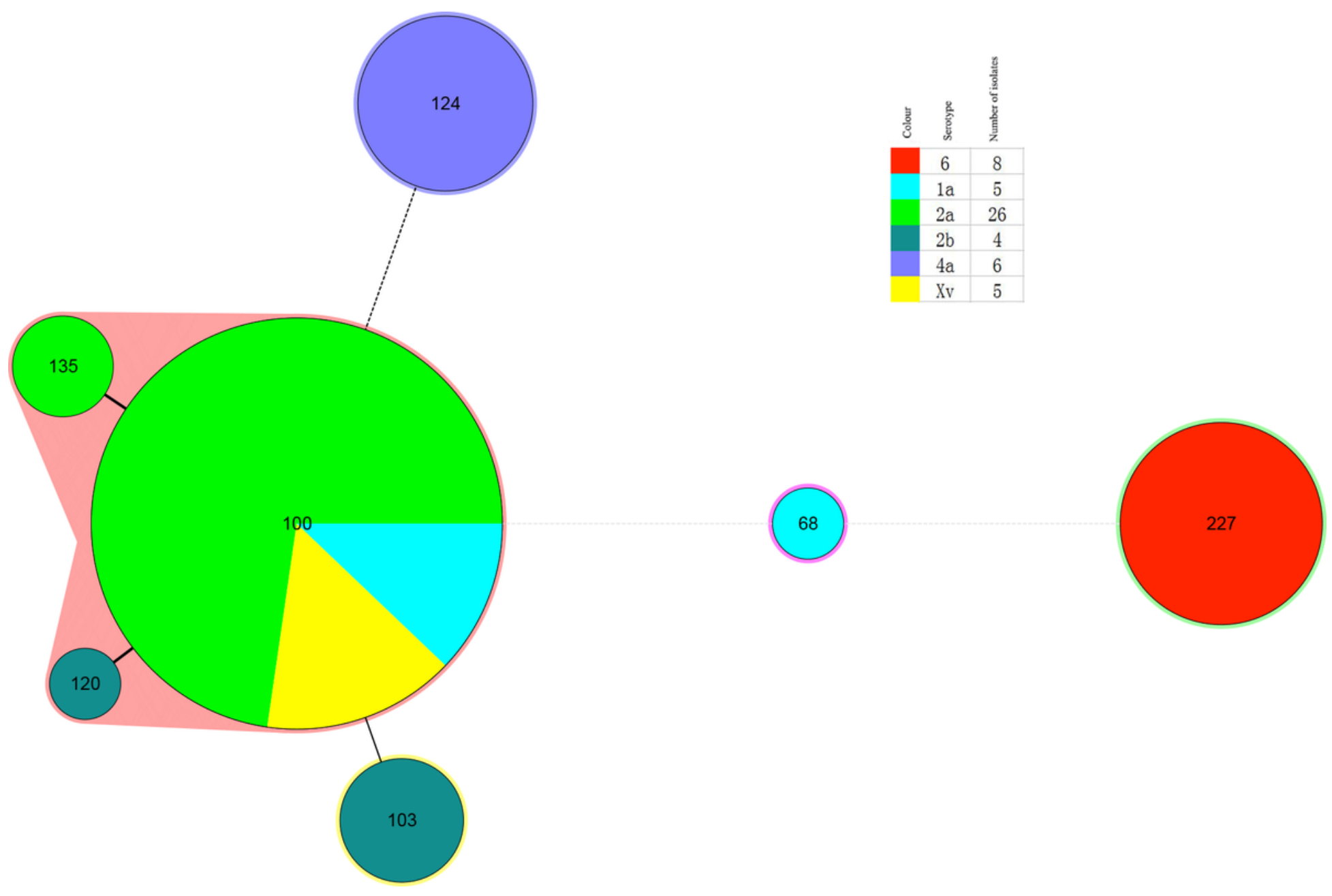

Figure 3

Minimum spanning tree of the $54 \mathrm{~S}$. flexneri isolates from diarrhea calves based on multilocus sequence typing (MLST). The minimum spanning tree was constructed using the 7 identified STs obtained from the 54 isolates using BioNumerics Software. Each circle corresponds to a single ST. The shadow zones in different colors correspond to different serotypes. The size of the circle is proportional to the number of isolates, and the color within the circles represents the serotype of the isolates. The corresponding color, serotype, number of isolates and background information are shown to the right of the minimum spanning tree. 


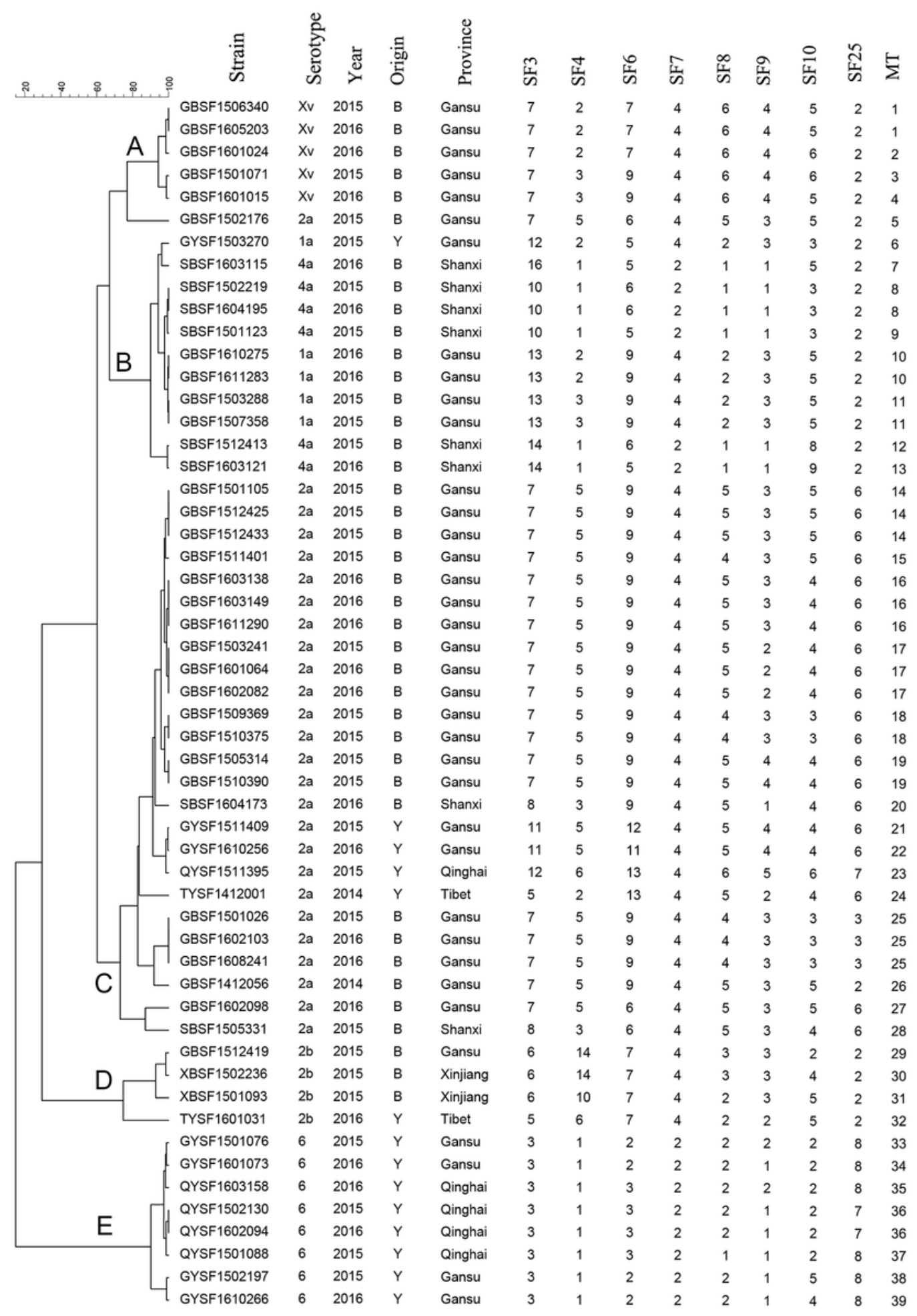

Figure 4

Relationship of S. flexneri isolates isolated from diarrhea calves based on MLVA. Isolates were analyzed using an eight VNTR loci MLVA scheme. The dendrogram was constructed using UPGMA. The corresponding MLVA type with the copy numbers of the eight VNTRs, serotype, and background information are shown to the right of the dendrogram. 


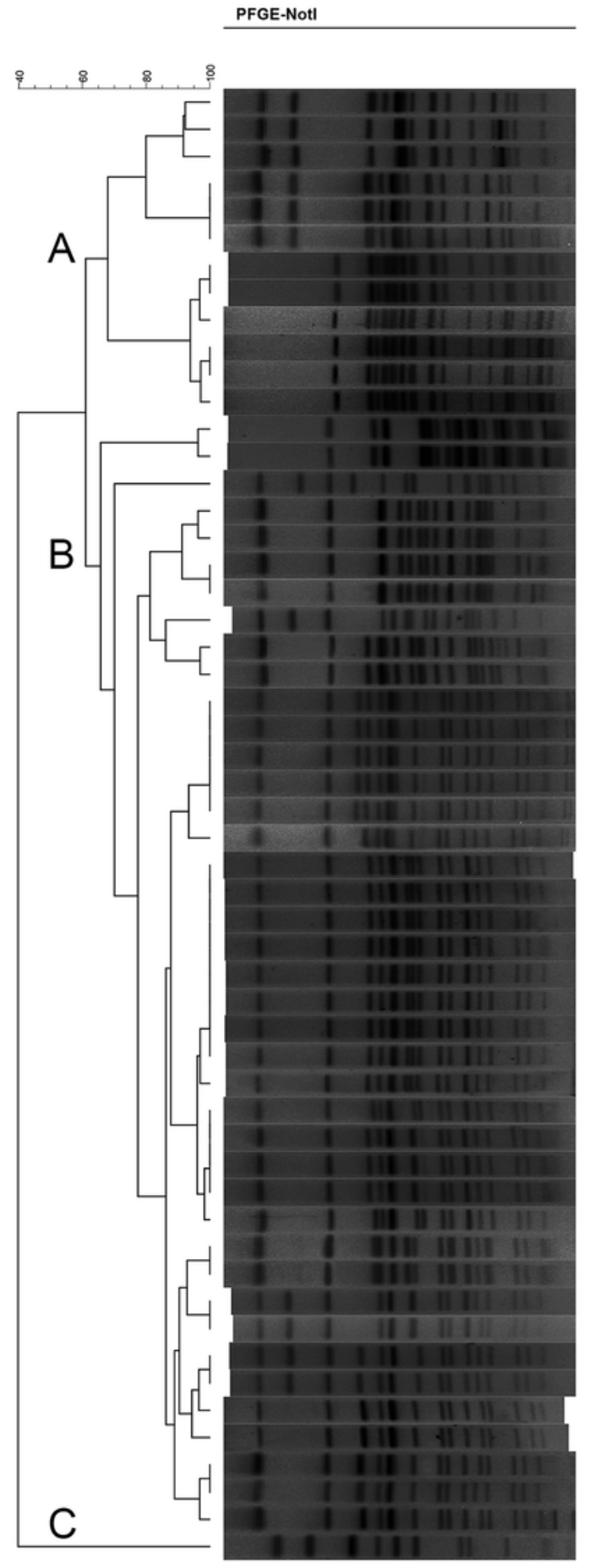

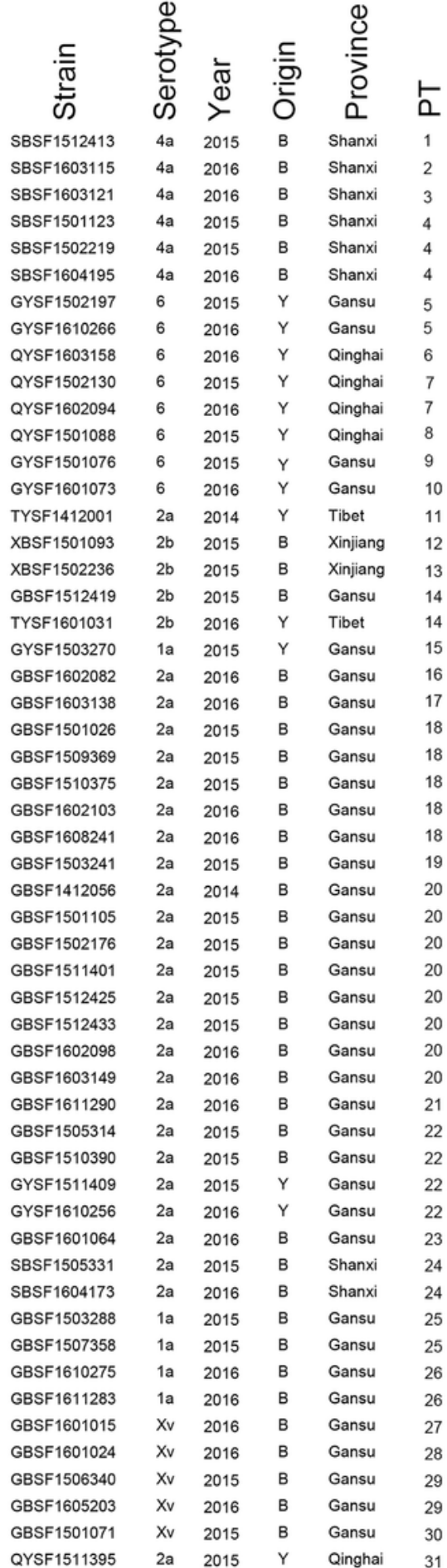

Figure 5

Dendrogram of 54 Notl-digested S. flexneri isolates based on the cluster analysis of PFGE patterns. The dendrogram was constructed using the UPGMA clustering method. The corresponding antibiotic resistance profile, PFGE pattern and background information for each strain are listed on the right side of the dendrogram. 
This is a list of supplementary files associated with this preprint. Click to download.

- TableS1.docx

- TableS2.docx 